\title{
MACRO PHOTOGRAMMETRY FOR THE DAMAGE ASSESSMENT OF ARTWORK PAINTED SURFACES
}

\author{
L. M. Angheluță ${ }^{1 *}$, R. Rădvan ${ }^{1}$ \\ ${ }^{1}$ INOE 2000, National Institute of Research and Development in Optoelectronics, 409 Atomiștilor, Măgurele, Ilfov, Romania \\ (laurentiu, radvan)@inoe.ro
}

Commission II, WG II/8

KEY WORDS: very close-range photogrammetry, polychrome, cultural heritage, guideline, long term monitoring, 3D digitization

\begin{abstract}
:
In this paper we are presenting our approach and workflow for macro photogrammetry as a complementary method for 3D digitization of polychromies on wood support. Macro photogrammetry can be used for detailed documentation of physical damages in painting layers, underlayers or support. For a better presentation of the context, a selection of typical physical damages, relevant to this study, are explained. Two case studies are presented. Two wooden icons realized with different techniques and presenting different types of physical deterioration. The experiments detailed within this paper represent the first epoch of long-term monitoring of the restoration interventions for each of these icons. All the stage of our workflow is thoroughly detailed with acquisition parameters and processing settings in order to better understand the results and where things can be improved. For each icon a full body photogrammetry process is described, followed by macro photogrammetry on smaller selected areas on the icons' surfaces. Macro magnifications of 1:1 and 2:1 are obtained by using a dedicated macro lens and extension rings. Lighting setups and focus stacking process are also described for a better understanding of the parameters and settings used.
\end{abstract}

\section{INTRODUCTION}

\subsection{Context of this study}

Cultural heritage assets are constantly exposed to a variety of risks. Whether it is the human hand (intentional - vandalism, unintentional - accidents) or natural phenomena and conditions (humidity, temperature, light or natural accidents), the integrity and quality of these goods is endangered without a good conservation plan. Many, if not the majority, of these risks can be prevented or at least controlled by preventive conservation measures (ICOM-CC membership, 2008).

A mandatory measure in the preventive conservation practice is the monitoring and control of the environment of the storage conditions. In controlled environments temperature, humidity, light and pollutants are adjustable parameters that can be artificially modified by man depending on the physicochemical properties and the state of conservation of the constituent materials and surfaces of the protected objects.

Museums and art galleries usually complement the environment monitoring with periodical imaging and physico-chemical documentation and investigations in order to establish the longterm conservation and/or restoration strategies.

While physico-chemical investigations deal with the chemical inspection of the surface layer's compounds (Ghervase et al. 2018), modern imaging documentation is about replicating in digital format the artwork's physical surface, structure and chromatic characteristics. There is a plethora of non-destructive imaging methods differentiated by the electromagnetic spectrum range where they actually record data: X-ray, ultraviolet (fluorescence), visible, near infrared, infrared etc. Each of these methods gives us different data about the quality, structure and artwork's history or about the artist's skill technique.
Three-dimensional digitization is also part of the imaging documentation process for artworks. This method is used to generate accurate digital 3D replicas of the artwork surfaces with geometric details of tens of micro-meters. It is usually employed by using active methods (like 3D laser scanning) or passive methods (photogrammetry).

While 3D digitization is intuitively used on monuments or "3D objects" it can also be quite useful for the documentation of planar surfaces of artworks like paintings (on different supports: walls, canvases, wood etc.). Photogrammetry documentation of artworks has been successfully reported in many other studies (Abate, 2019). One application is to monitor physical deformations of the support structure of large paintings during long time restorations processes and thus preventing further possible damage (Robson et al. 2004). Comparing 3D reconstructions realized at different periods of time the restorers can easily detect and adjust any physical deformations that have inferred to the artwork during their work. Other reports include under layer 3D reconstruction based on multispectral images photogrammetry (Grifoni et al. 2017).

In this paper we are using a photogrammetric method for the accurate documentation of physical damages into painting surface layers using macro photography and consequently macro photogrammetry (or very close-range photogrammetry).

Employing macro photography raises several problems like getting too close to the surface, the reduced the amount of light that the camera receives and the much thinner depth of field, but nevertheless it can be used for 3D digitization purposes (Gajski et al. 2016). A relevant study for this work comes from the field of forensics where very close-range photogrammetry was used to measure and characterize the micromorphology of cut marks in bones (Gonzalez et al. 2015). 


\subsection{Physical defects specific to polychrome surfaces}

By polychrome surfaces we understand artwork surfaces that are decorated with different colours. These surfaces may be on different supports (wall, metal, wood, canvas, paper etc.), on architectural elements, sculptures, pottery etc.

Polychrome surfaces are composite structures where complex reactions take place between the different compounds. Their deterioration may be generated by:

materials (pigments) and the technique used,

ageing of pigments,

environment,

weariness and mechanical deformations.

Besides these problems, related to the nature and the painting technique, the conservation status of polychrome surfaces can also be influenced by other factors like:

- the painting support,

- storage conditions (light, humidity, dust, biological attacks, insect etc.),

past restoration interventions,

- accidents.

Painting support is strongly affected by the changes in temperature and relative humidity. These changes, combined with infiltration, usually lead to the dilatation or contraction of wood and fabric materials. Such variations generate a wide range of defects in the painting layers (Weyer et al, 2015).

Only a short selection of such defects are going to be explained, that are relevant for the measurements included in this paper.

Cleavage is a type of defect where the layers of the paint film are lifting or separating from the ground or from other painting layers. This effect can lead to blisters, wrinkles or flaking (if cracks are present). Cracks (or fissures) appear as separations in the paint layer, ground or even support, which are perpendicular to the surface of the painting. These can be classified as stress cracks (due to physical pressures on the painting layer), mechanical cracks (radial) or ageing cracks. A network of such cracks over a larger area of the painting is known as craquelure.

Other types of defects are resulted from erroneous physical manipulation of the painting support. These have immediate effect and can result in: creases of the canvas, deformations, contortions, ruptures or cavities.

Insect damage is another common type of physical defect. Many species feed on the painting materials producing damage. Visible signs are the tunnels in the wood or holes in the fabric.

Due to their size, all these types of damage require macro photography for proper documentation and further monitoring. For 3D digitization, photogrammetry is still the best choice in this case for several reasons. The competing methods for 3D reconstruction are structured light scanners and 3D laser scanners. Both these technologies produce high precision measurements and 3D reconstructions but with a significant financial effort. A great disadvantage of these methods is the lack of high-quality textures without the possibility of real colour correction. Chromatic quality is an essential aspect of long-term monitoring of the preservation status of polychrome surfaces. The financial aspect is another factor that weighs in the favour of photogrammetry.

\section{MACRO PHOTOGRAMMETRY WORKFLOW}

\subsection{Preliminary observation and workspace setup}

In this first stage, the surface is visually analysed noting relevant observations regarding manipulation fragility, physical defects and regions of interest. The object/surface is photographed full size on all sides, with scales and colour and white balance markers.

Workspace management is critical. The first priority is the object/surface integrity so the working area should be cleared of all unnecessary clutter and establish the subject (if movable) and all other equipment positions. Next, the lighting arrangement should deliver a constant level of illumination all over the surface. Light source positioning must eliminate any sort of shadows on the surface and allow at the same time a fast shutter time. In macro photography setups it is recommended the usage of non-heating light sources.

Also, during this stage, the data acquisition parameters are decided. The casuistry, the spatial resolution needed and the available optical equipment (lens focal length, sensor size) determines the camera working distance, images vertical and horizontal overlap percentage, the number of images to be recorded, camera parameters, the incident angle of photography (oblique or perpendicular) and scaling targets positions.

An important aspect that must be accounted for is the means of camera position displacement on three axes (vertical, horizontal and depth). The available tools (manual or motorized sliders) and their displacement ranges dictates the size of the area on the surface that can be recorded. Graded sliders and tripods are required for high quality results.

\subsection{Data acquisition}

Once the workspace, light sources and camera montage are all setup, the camera and lens parameters can be set accordingly to the observations made in the first stage. For a correct chromatic representation white balance and colour checker cards are mandatory.

It is recommended to record the images in RAW format for colour, shadow and exposure corrections in post processing. A good file management strategy is a great way to avoid data loss and ease of access over time, especially in cases of long-term monitoring of the same subject with lots of epochs.

An important step after the image acquisition is the postprocessing. Qualitative free and commercial image editing software programs allow batch operations that saves a lot of time. Usual operations are: lens correction, white balance, colour profile and over / under exposure adjustments.

In macro photography most often the depth of field is much smaller than the depth of the object. Therefore, the focus stacking technique is used in order to create an in-focus image from several images focused at different distances.

Edited images are saved on disk in JPEG or TIFF format, ready to be imported in 3D reconstruction software. 


\subsection{D reconstruction}

Digital photogrammetry is back in the business and more than ever now is accessible to larger interested groups. Commercial and free software solutions are currently competing for automated, fast and reliable processing software packages.

Like in photography, in 3D reconstruction the best solution is the one that is already available to you. In this regard, there are several free or open source software solutions for photogrammetric 3D reconstruction processing each with its advantages and disadvantages (Remondino et al. 2017).

\subsection{Deliverables creation}

Typical deliverables can have many forms: digital 3D files, DEMs, orthophoto mosaic, complex visual system for data corroboration or physical 3D prints.

The resulted 3D model is exported in a convenient 3D format (OBJ, PLY, STL etc.) and is prepared for post-processing: polygon simplification, re-topologisation and texture baking. DEMs or orthophotos can be directly generated by the photogrammetric processing software. For 3D printing the mesh must be simplified to a compatible number of polygons and made watertight.

For long term monitoring tools like QGIS can be of great value. Its database can be used for storing and associating complementary imaging data like photography, radiographs, multi and hyperspectral data, spectroscopy data and of course, 3D data.

\subsection{Presentation}

The final act in the 3D digitization process is the presentation or the publication of the deliverables. Depending on the purpose of the digitization, the deliverables can be presented to the beneficiaries in several ways: final digital 3D files (mesh or point cloud), detailed technical reports, database with multiple imaging data and/or at periodic epochs (QGIS), 3D prints, artistic video clips or $2 \mathrm{D}$ renders.

3D file formats are not so user friendly without specific visualization solutions, unlike photos, videos or other document files. Luckily today there are several online services that allow, freely, the upload and view customization of many 3D formats or embed them in custom web pages like virtual museums and galleries. This is the easiest way to publish the 3D content fast and worldwide accessible.

\section{3D DIGITIZATION OF PAINTED WOOD PANELS}

This workflow was tested on two icons painted on wooden panels. The equipment used in both cases was a full frame body Nikon D810 with Nikon AF-S Micro Nikkor 60 mm 1:2.8G ED. This lens has 1:1 reproduction factor and in macro mode its length is fixed, an important factor for working close to the surface. For the detailed documentation of smaller areas extension rings $(68 \mathrm{~mm})$ were used to achieve 2:1 magnification, resulting in focus stacking acquisition and post-processing.

\subsection{St. Constantine and Helen icon}

The first icon digitization was part of a larger pre-restoration documentation that included X-ray imaging, hyperspectral and multi spectral imaging that is going to be followed by repeating these same imaging methods after the restoration, including physico-chemical analysis (XR-F and FTIR spectroscopy).

This icon is one of four set of icons from St. Nicholas wooden church in Şirineasa, Vâlcea county, that are part of the larger study. The painting is dated to the middle of the XIXth century, but raking light photography and X-ray imaging proved that there is an older painting under the visible layers.

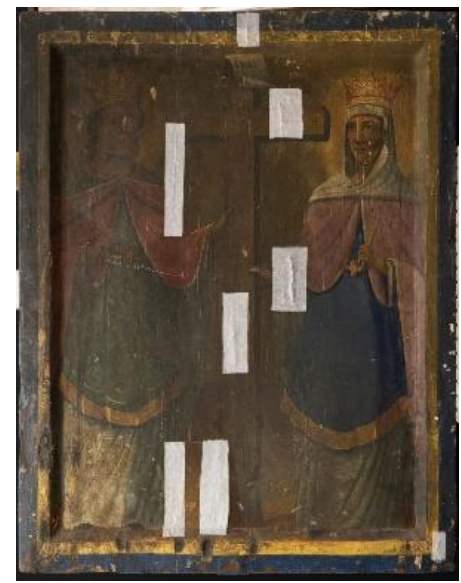

Figure 1. St. Constantine and Helen icon, painted on wood. Corrected image

The aim of the 3D digitization in this case was a detailed full body recording and macro-documentation of two areas that presented typical surface damages. The first area perimeter contains a portion were the paint layer is lifted a few millimetres creating a cleavage, and small cracks. The second area is a typical blistering surface, with many bubbled shape cleavages. Many of these blisters have missing paint layers caused by the flaking (peeling) effect, making the under-painting layers visible.

3.1.1 Preliminary observations. In this first step a photographic documentation was realized on both sides and all edges. The size of the subject was $49 \mathrm{~cm} \times 64.5 \mathrm{~cm}$ x $3.7 \mathrm{~cm}$. The edges of the icon are embossed resulting a $0.5 \mathrm{~cm}$ level difference from the body of the painting. The full body digitization parameters had to account for that difference in order to avoid defocused areas in the images set.

The subject was fixed on a table easel in vertical position. The camera was mounted on a solid and accurate positioning system (ARTIST CPS-200 from ArtInnovation) for a smooth and precise $\mathrm{X}-\mathrm{Y}$ camera displacement. The light sources were two portable LED lamps mounted on articulated arms on each side of the camera, to the positioning system stand. This way it was ensured the same lighting conditions in all the images.

For macro shots, a manual macro $\mathrm{X}-\mathrm{Y}$ rail was used for camera precise displacement and manual focus.

Working distance can be either measured from the sensor symbol on the camera body or calculated by adding focal flange distance (distance between the sensor plane and the lens mounting plane) with lens physical length and the distance between the lens and the subject surface.

For the full body capture, the working distance (measured from the sensor position symbol on the camera to the subject) was 31.5 $\mathrm{cm}$ to the embossed edge (respectively $32 \mathrm{~cm}$ to the body of the painting). With the lens focused at $31.5 \mathrm{~cm}$ and a lens aperture of 
$\mathrm{f} / 8$, the level difference was within the calculated focal depth of field $(1 \mathrm{~cm}$ starting at the focused point).

The camera was set to shoot RAW files at ISO 100 and $1 / 4 \mathrm{~s}$ exposure time and $f / 8$ lens aperture (best quality image for this lens).

For macro capture the working distance was $22,7 \mathrm{~cm}$ (distance between the lens and surface was $2.4 \mathrm{~cm}$ ). Magnification was close to $2: 1$. Even with the lens aperture closed to $f / 18$ there were frames that needed focus stacking. In this case the ISO was increased to 800 in order to keep a reasonable exposure time at $1 / 5 \mathrm{~s}$.

A laptop with a remote camera control software was used for visual feedback of camera positioning and precise focus. This method also prevented any risks regarding the motion blur effects caused by camera micro-shaking during a physical shutter press.

3.1.2 Data acquisition. White balance and colour checker targets were captured at the same working distance and lighting conditions in order to correct the image set in post-processing for realistic texture. A scale bar was included during the images set for size adjustments and precise measurements.

For the full body digitization, a total of 1176 images were recorded (including white balance and colour checker shots) using a planar camera displacement strategy with perpendicular orientation to the surface. All the shots were parallel.

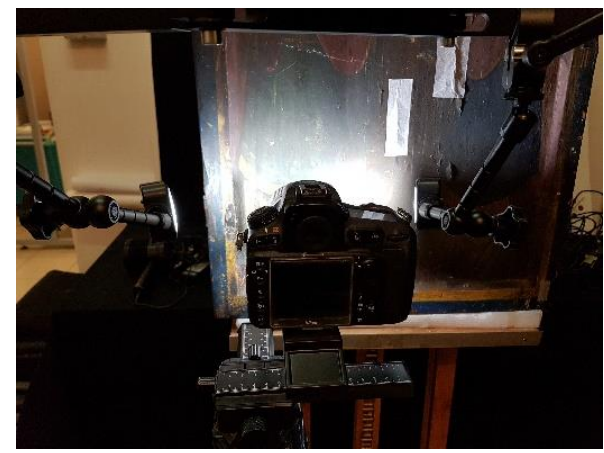

Figure 2. Macro shooting lights and camera setup

The real size area captured by one image was $\sim 11 \mathrm{~cm} \mathrm{x} 7 \mathrm{~cm}$ and the camera displacement step was $1.5 \mathrm{~cm}$ on horizontal and $1 \mathrm{~cm}$ on vertical. The resulting overlapping factor was $\sim 86 \%$ on both horizontal and vertical axes so that all the small sized details would have enough coverage in order to be reconstructed.

For the macro capture were selected two smaller areas that were representative for specific physical damages in the painting layers. For the first area were recorded 221 images while for the second area 215 images. The areas with severe physical damage focus stacked images were recorded.

The recorded images are saved on disk in specific and identifiable folders. With the colour checker image, a camera profile is generated and then used in colour adjustment of the images. Using an image editor that allow batch adjustments the following general edits were performed: colour adjustment, white balance adjustment and lens profile correction. Finally, images are exported in a separate folder in JPEG format, at maximum quality and 300 dpi.

An important aspect for macro recordings is the accurate adjustment of the positioning system in relation to the subject surface. The camera must slide on perfectly parallel directions (on both axis) otherwise the working distance changes, resulting in defocused images and different spatial resolutions. In this regard the distance to the surface was measured at the starting and ending positions of the camera.

3.1.3 3D reconstruction. The software package used in this project is Agisoft Metashape (Photoscan). This program splits the 3D reconstruction process in several stages: image alignment (eventually with masking), dense point cloud generation, mesh generation and mesh post processing and export.

For the full body reconstruction, image alignment was processed at highest quality with 10.000 tie points limit and 40.000 key points limit using adaptive camera model fitting. There was no need for masking. All the images were aligned resulting a 1.5 million point sparse cloud. There were used 4 reference markers and two scale bars.

The dense cloud was generated also on the high setting resulting 505 million points. This dense point cloud was used to generate the 3D mesh. A Python script for multi-chunk processing was used. The dense cloud was split in 4 chunks with $10 \%$ overlap and merged back in an 83 million polygons mesh.

The reconstructed mesh was simplified to 2 million polygons, suitable for export, online publishing or 3D PDF export.

The first macro area was processed with the following parameters: image alignment at highest quality (443 million points), dense point cloud at ultra-high quality (1.2 million points) and the mesh was generated using the split in chunks script with 6 chunks and $10 \%$ are overlapping (263.6 million polygons). The mesh was simplified to 2 million polygons. The focus stacked images were not stacked in a unified image, but were processed as they were in order to test the processing program capacity of discrimination the out of focus areas.

The second macro area was processed with the same parameters as the first one. The resulted sparse cloud has 0.5 million points, the dense cloud 1.4 million points and the reconstructed mesh had 49 million polygons (simplified to 2 million).

Texture in all three cases was generate at $8 \mathrm{~K}$, contributing to high resolution orthophoto.

\subsection{St. Nicholas icon}

This is an icon from a private collection and is in a critical state of degradation with strong cleavages, cracks and craquelures. It was realized using a non-conventional, experimental, method: gypsum applied directly on wood and then painted over.

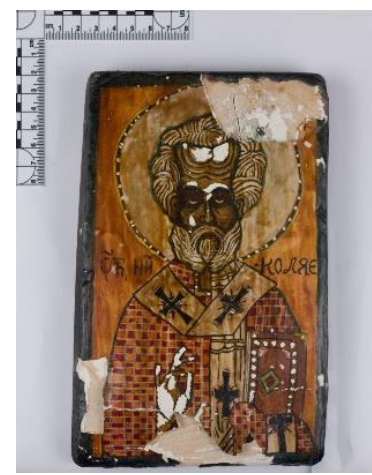

Figure 3. St. Nicholas icon with scale. Colour corrected image 
The aim of the $3 \mathrm{D}$ digitization in this case was a detailed full body digital reconstruction and a macro 3D documentation with two different magnifications of the same area. The selected area presents loss of paint layers and fine craquelures.

3.2.1 Preliminary observations. First thing to notice about this subject is its critical condition and fragility. A photographic documentation was realized. The size of this subject is $21.3 \mathrm{~cm} \mathrm{x}$ $13 \mathrm{~cm} \times 2.8 \mathrm{~cm}$. The painting layers in the cleaved areas was lifted up to $1 \mathrm{~cm}$ from the wood panel. This level difference had to be accounted for during the full body digitization.

The icon was fixed on a table easel with a marked grid panel behind. The purpose of the grid was both for scale and linearity control of the camera horizontal displacement. The camera was mounted on a special tripod head that allows precise and smooth vertical displacement on a graded slider. On top to the vertical slider the X-Y macro rail was mounted with the camera. This set up allowed us precise sliding control on all three axes: $30 \mathrm{~cm}$ on vertical and $15 \mathrm{~cm}$ for lateral and depth displacements.

Lighting sources were two sets of $5 \mathrm{x} 45 \mathrm{~W}$ white fluorescent lamps enclosed in softboxes, on separate stands. While this setup was satisfactory for full body digitization, for macro shooting the big size of the softboxes complicated the camera displacement.

For full body digitization the working distance was established at $23 \mathrm{~cm}$ (meaning $2.7 \mathrm{~cm}$ distance between lens and surface). Camera parameters were set to RAW shooting, ISO 640, f/9 and an exposure of 1/30s. Resulted depth of field was $0.6 \mathrm{~cm}$ which resulted in multiple stack shots for areas with pronounced layer lifting.

A single area of $6 \mathrm{~cm} \mathrm{x} 4 \mathrm{~cm}$ was selected for 1:1 and 2:1 macro photogrammetry capture, with the sole purpose of results comparison. The working distance was set at the minimum focusing distance of the lens $(1.9 \mathrm{~cm})$. For 1:1 shooting, camera parameters were set to ISO $640, \mathrm{f} / 9$ and an exposure of $1 / 60 \mathrm{~s}$. But the lighting sources were brought much closer to the surface. For $2: 1$ shooting, camera parameters were set to ISO $640, \mathrm{f} / 4.5$ and an exposure of $1 / 50 \mathrm{~s}$. With the lighting sources in the same spots.

Like in the first case, a laptop was used for remote control and visual feedback.

3.2.2 Data acquisition. White balance and colour checker targets were captured once in the lighting conditions for the full body digitization. A scale bar was used only for the full body, while for the macro shoots, because of the fragility of the surface, distances between recognizable physical references on the surface were measured.

Full body took 372 images using planar camera displacement with perpendicular orientation to the surface. Camera displacement step was $1.5 \mathrm{~cm}$ on horizontal axis and $0.5 \mathrm{~cm}$ on vertical axis. The real size area captured in one image was $16 \mathrm{~cm}$ $\mathrm{x} 11 \mathrm{~cm}$.

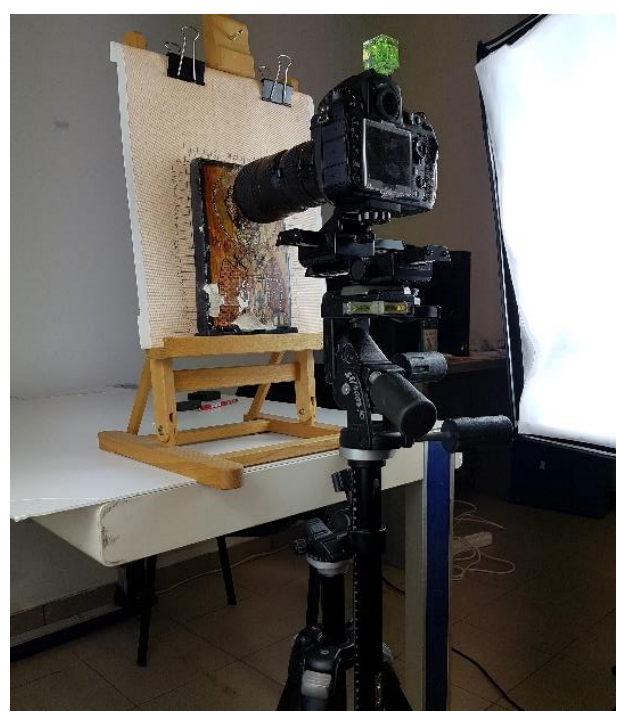

Figure 4. Setup for macro shooting. Vertical graded slider and horizontal X-Y graded slider.

For 1:1 macro image set were recorded 218 images. Areas with great layer displacement required multiple focusing positions (stacks of 3 to 7 images). After the focus stacking processing the final number of images was 116.

For 2:1 macro image set were recorded 132 images with even more stacks per frame. The final number of images after the focus stacking process was 44 . Unfortunately, many of these resulted images showed partial blurred areas, indicating that more photos at different focusing steps should've been recorded. This resulted in poor $3 \mathrm{D}$ reconstruction.

The image processing (colour and white balance adjustments, lens profile correction) was realized before the focus stacking. The focus stacking was realized using Helicon Focus Pro and its plugin for Adobe Lightroom Classic. This process is quite straight forward: in Lightroom the stack image set is selected exported to Helicon Focus, processed there and then the result is saved to disk. This workflow saves a lot of time during the image processing stage.

3.2.3 3D reconstruction. The same software parameters were used for all the processing in this second case. For full body reconstruction, the image alignment generated a sparse point cloud of 0.38 million points, dense point cloud (high setting) had 41.7 million points and the 3D model was reconstructed with 116 million polygons, simplified to 2 million polygons. There were used three reference markers and one scalebar.

For 1:1 macro image set, all the 116 were aligned at the highest quality setting resulting 0.25 million points. The point dense cloud was generated using ultra-high quality setting and 467 million points were resulted. The 3D model reconstructed had 304 million polygons and was simplified to 4 million.

All images in the 2:1 macro image set were aligned. Dense point cloud was generated using ultra-high quality, resulting 471 million points. While the surface was thus reconstructed, major errors caused by bad image quality (not enough focus stacking) made this reconstruction (107 million polygons) unusable. 


\section{RESULTS AND DISCUSSION}

In both cases were created the same type of deliverables: OBJ format 3D files, orthophotos, DEMs and interactive online presentations (in Sketchfab).

St. Constantine and Helen icon full body 3D digitization was successful. A 31358x39485 pixels orthomosaic was generated using the 3D mesh processed, with a $0.0176 \mathrm{~mm} /$ pixel resolution. The DEM was also generated based on the 3D model, with $25871 \times 31294$ pixels and $0,0353 \mathrm{~mm} /$ pixel resolution.
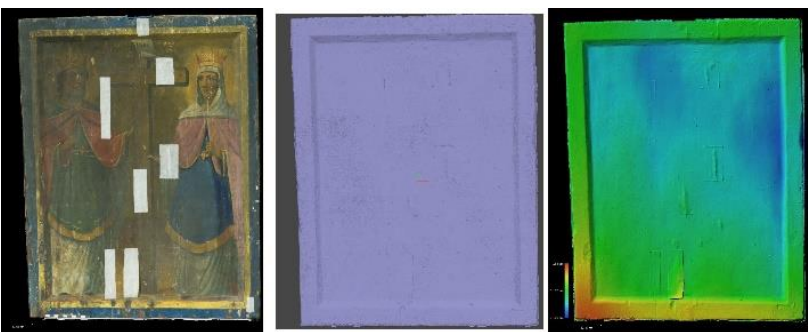

Figure 5. Left: orthophoto mosaic. Center: Mesh. Right: DEM

The 3D digital reconstruction was exported to OBJ format file with an $8 \mathrm{~K}$ realistic colour texture. For an easier access to different experts, the model was also uploaded to an online interactive $3 \mathrm{D}$ medium.

The first area digitized using macro photogrammetry was successfully processed. The level of detail compared with the same area of the full body digitization was much increased. The resulted DEM has a $1.63 \mu \mathrm{m} /$ pixel resolution.

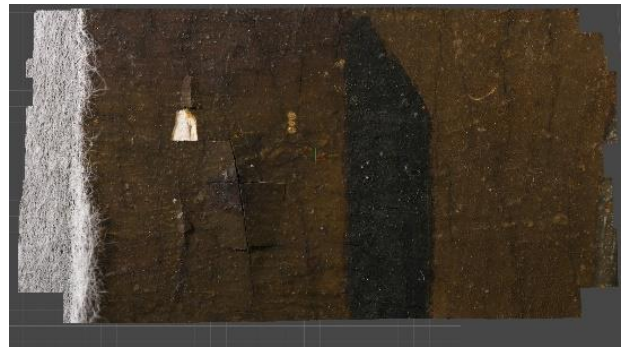

Figure 6. St. Constantine and Helen: textured 3D model reconstructed using macro photogrammetry (Area 1)

This area included liftings, cracks in the painted layer and a small area of loss. In the DEM these areas are easily marked and measured.

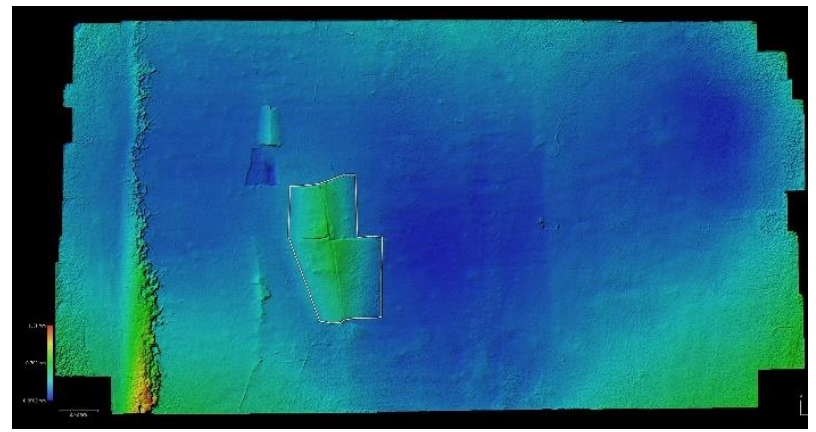

Figure 7. St. Constantine and Helen: DEM with marked lifted layer (Area 1)

As mentioned in the preliminary observations stage, the second area is characterized by bubble shaped blisters and flaking that reveals the layers of another painting underneath.

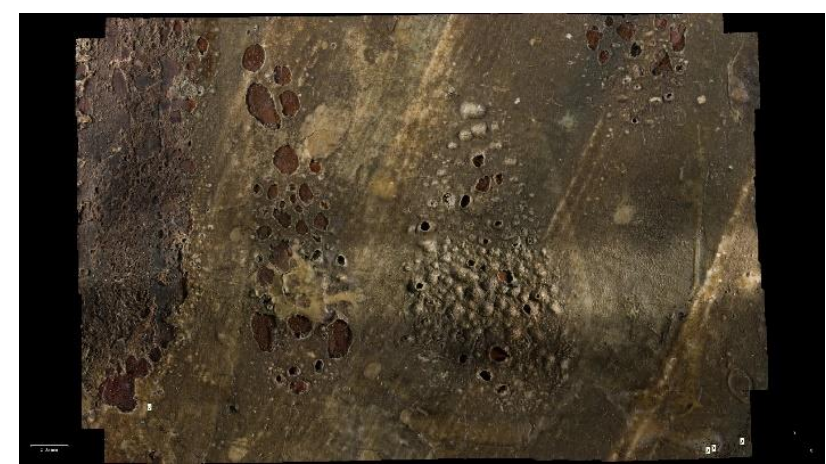

Figure 8 . St. Constantine and Helen: area characterized by blisters and flaking. Orthomosaic

The reconstruction of this area was also successful, minus a lighting error during several shots that resulted in a band of increased luminosity in the texture (see Figure 8 ). This can easily be corrected in careful image post processing.

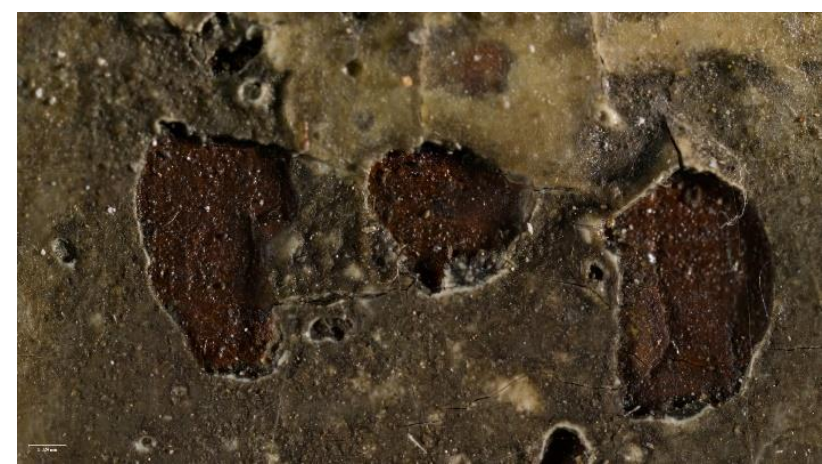

Figure 9. 6-millimetre-wide image crop from the orthomosaic of the second area

St. Nicholas icon full body 3D digitization was also successful. The orthomosaic was generated with $7646 \times 10723$ pixels and $0.0189 \mathrm{~mm} /$ pixels. The DEM was also generated based on the 3D mesh, with a resolution of $4909 \times 5903$ pixels and 0.0387 $\mathrm{mm} /$ pixel.

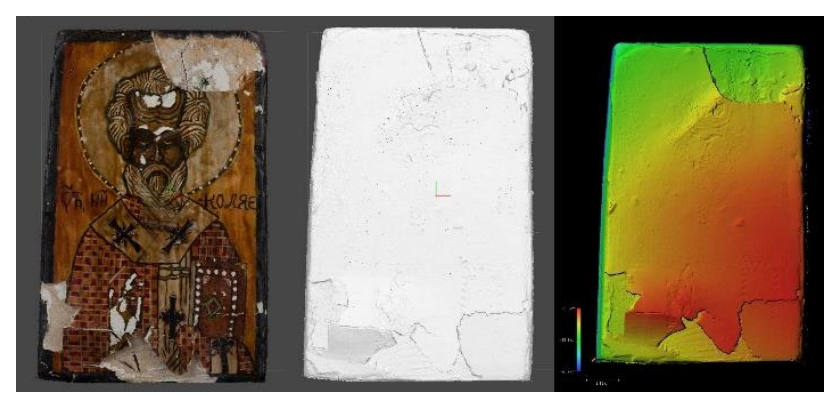

Figure 10. St. Nicholas., full body photogrammetry. Left:

Textured 3D model. Center: Mesh. Right: DEM

Similar deliverables with the first icon, were produced and exported.

As mentioned, a single area was selected for macro photogrammetry. Two data acquisitions were performed with different optical magnifications 1:1 and 2:1.

Data acquired at 1:1 magnification had no problems during processing and 3D reconstruction, with only several frames that 
required focus stacking. The 3D model resulted offered better details regarding the craquelures.

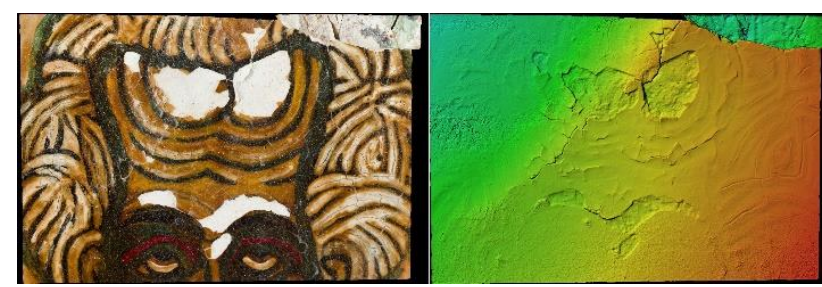

Figure 11. St. Nicholas, selected area for macro photogrammetry. Left: textured 3D model. Right: DEM

For each 3D model processed, the scale precision was verified and adjusted by measuring recognizable features on both the object surface and the digital reconstruction.

\section{CONCLUSION}

The experiments in this study were conducted following a precise workflow. The purpose of this paper was to put some light onto the use of macro photogrammetry and whether this method can actually be used or not for assessing different types of physical and chromatic damage in the polychrome surfaces. Our results show that reliable data can be generated from macro magnifications of $1: 1$ and $2: 1$. The critical reduction of the depth of field requires careful focus stacking. For greater magnifications, beyond 2:1, an automated focus stacking rail is definitely recommended in order to avoid the unavoidable human errors that occur at this level of precision.

Macro photogrammetry data processing and 3D model reconstruction did not pose any difficulties with the selected software package, as long as the images are perfectly focused.

At key epochs during the restoration process of these icons, other sets of data will be acquired and processed. Orthomosaics and DEMs together with complementary imaging data (multispectral, hyperspectral or radiographs) but also physico-chemical will contribute to a complete QGIS package that can be extremely useful during the restoration and consolidation process of the icons.

\section{ACKNOWLEDGEMENTS}

This study is financed by the Ministry of Research and Innovation through Program I - Development of the National research-development system, Subprogram 1.2 - Institutional Performance - Projects funding excellence CDI, Contract no.19PFE/17.10.2018 and "Program Nucleu" PN 19-18.01.01: OPTRONICA VI

\section{REFERENCES}

Abate, D., 2019. Documentation of paintings restoration through photogrammetry and change detection algorithms. Heritage Science. DOI: 10.1186/s40494-019-0257-y.

Gajski, D., Solter, A., Gasparovic, M., 2016. Applications of macro photogrammetry in archaeology, ISPRS - International Archives of the Photogrammetry, Remote Sensing and Spatial Information Sciences. Vol. XLI-B5, pp. 263-266.

Ghervase, L., Cortea, I. M., Rădvan, R., Ratoiu, L., Chelmuş, A., 2018. Complementary investigations of two Lipovan-style icons, Microchemical Journal, Vol. 138, pp. 509-518.
ICOM-CC, Terminology to characterize the conservation of tangible cultural heritage, 2008. $15^{\text {th }}$ Triennial Conference, New Delhi, 22-26 September 2008.

http://www.icom-cc.org/54/document/icom-cc-resolutionterminology-english/?id=744\#.XSd_m-gzaUk (11.07.2019)

Gonzalez, M. A. M., Yravedra, J., Gonzalez-Aguilera, D., Palomeque-Gonzalez, J. F., Domínguez-Rodrigo, M., 2015. Micro-photogrammetric characterization of cut marks on bones, Journal of Archaeological Science, Vol. 62, pp. 188-144.

Grifoni, E., Legnaioli, S., Lorenzetti, G., Pagnotta, S., Palleschi, V., 2017. Image based recording of three-dimensional profiles of paint layers at different wavelengths, European Journal of Science and Theology, Vol. 13, 2, pp. 127-134

Remondino, F., Nocerino, E., Toschi, I., and Menna, F, 2017. A critical review of automated photogrammetric processing of large datasets, Int. Arch. Photogramm. Remote Sens. Spatial Inf. Sci., XLII-2/W5, pp. 591-599 https://doi.org/10.5194/isprs-archives-XLII-2-W5-591-2017.

Robson, S., Bucklow, S., Woodhouse, N., Papadaki, H., 2004. Periodic photogrammetric monitoring and surface reconstruction of a historical wood panel painting for restoration purposes, ISPRS Archives - Vol. XXXV Part B5, pp. 395-400.

Weyer, A., Picazo, P.R., Pop, D., Cassar, J., Özköse, A., Vallet, J.-M., Srša, I. (Ed.), 2015. EwaGlos - European Illustrated Glossary of Conservation Terms for Wall Paintings and Architectural Surfaces. Michael Imhof Verlag, Petersberg, Germany, 448p. ISBN 978-3-7319-0260-7 